\title{
Estrategias de \\ enseñanza aprendizaje \\ en el proceso formativo de estudiantes sordos \\ en la educación superior
}

Teaching strategies learning in the training process of deaf students in higher education

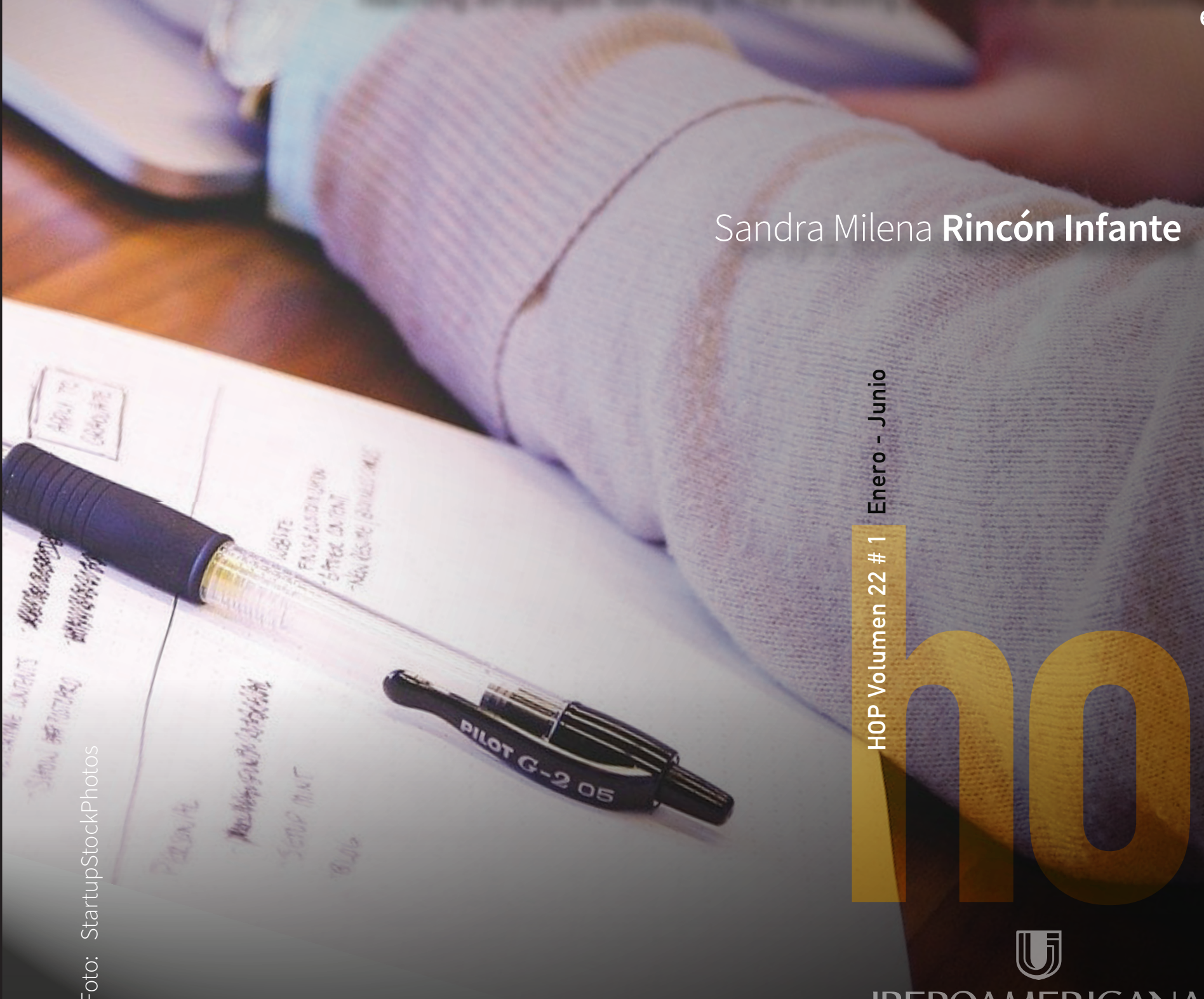

IBEROAMERICANA CORPORACIÓN UNIVERSITARIA
HORIZONTES PEDAGOGICOS ISSN-l: 0123-8264 | e-ISSN: 2500-705X 


\section{HORIZONTES PEDAGÓGICOS}

ID:

0123-8264.hop.22101

Title: $\quad$ Teaching strategies learning in the training process of deaf students in higher education

Título: Estrategias de enseñanza aprendizaje en el proceso formativo de estudiantes sordos en la educación superior

Alt Title / Título alternativo:

[en]: $\quad$ Teaching strategies learning in the training process of deaf students in higher education

[es]: $\quad$ Estrategias de enseñanza aprendizaje en el proceso formativo de estudiantes sordos en la educación superior

Author (s) / Autor (es):

Rincón Infante

Keywords / Palabras Clave:

[en]: Inclusive education; educational intervention; deaf community; strategic learning; communication; training; universal design

[es]: $\quad$ educación inclusiva; intervención educativa; comunidad sorda; aprendizaje estratégico; comunicación; formación; diseño universal

Proyecto / Project:

El aprendizaje estratégico como eje central en la inclusión de estudiantes sordos en la educación superior. Diseño de una intervención educativa.

Submited: $\quad$ 2020-02-07

Acepted: $\quad$ 2020-04-07

\section{Resumen}

Esta investigación tuvo como objetivo proponer una intervención educativa basada en el diseño

universal de aprendizaje - DUA, para fortalecer las prácticas pedagógicas inclusivas con un grupo de estudiantes sordos en una institución de educación superior en Bogotá - Colombia. En este sentido, se establecieron tres variables de análisis:

las estrategias de enseñanza que los docentes utilizan en su práctica pedagógica, las estrategias de aprendizaje que utilizan los estudiantes sordos y el rol del intérprete en el proceso de formación.

Teniendo en cuenta que el identificar dichas estrategias llevaría al docente a reflexionar sobre su práctica y establecer que ajustes y diseños metodológicos puede implementar en el aula, basados en los intereses y necesidades de los estudiantes, para garantizar su aprendizaje,

permanencia y promoción. Las características de la investigación propiciaron que el desarrollo metodológico se diera a partir de un enfoque cualitativo interpretativo, a través de un diseño de investigación acción, empleando diferentes métodos; tales como observación participante y no participante, entrevistas semiestructuradas y grupos focales. Los resultados obtenidos son muestra del proceso de interpretación de los datos,

el procedimiento se basó en la lectura sistémica y profunda de cada una de las notas de campo, de las entrevistas y de los grupos focales, al igual que la visualización de los videos y su respectiva transcripción; para el análisis y sistematización de la información se hizo uso del software ATLAS.ti ${ }^{\circledR}$

(8.0) en su versión de prueba. Se logró establecer las características particulares del proceso de enseñanza- aprendizaje de los estudiantes sordos, las cuales están relacionadas directamente con aspectos motivacionales y con la posibilidad de comunicación con sus pares y docentes sin la mediación del intérprete, por otra parte determinar las estrategias de enseñanza empleadas por los docentes en un aula inclusiva, desde su experiencia individual, para así finalmente definir un enfoque pedagógico, objetivos, fases y evaluación de una

intervención educativa orientada a promover el aprendizaje estratégico en el proceso de formación profesional del estudiante sordo.

\section{Citar como:}

Rincón Infante, S. M. (2020). Estrategias de enseñanza aprendizaje en el proceso formativo de estudiantes sordos en la educación superior. Horizontes Pedagógicos, 22 (1), 1-12. Obtenido de: https://horizontespedagogicos.ibero.edu.co/article/view/1772

Lic Sandra Milena Rincón Infante, MEd sp

ORCID:

0000-0003-1754-6221

Source | Filiacion:

Universidad Internacional Iberoamericana

BIO:

Magister en Educación con Especialidad en Educación Superior de la Universidad Internacional Iberoamericana - UNINI - de Puerto

Rico. Licenciada en educación básica con énfasis en educación especial de la Universidad Pedagógica Nacional de Colombia.

City | Ciudad:

Puerto Rico [pr]

e-mail:

educadora.rehabilitacion@gmail.com 


\section{Estrategias de enseñanza aprendizaje en el proceso formativo de estudiantes sordos en la educación superior}

Teaching strategies learning in the training process of deaf students in higher education Sandra Milena Rincón Infante

\section{Introducción}

En Colombia, en el marco de la política de "Educación para Todos - EPT” (Fiske \& Peppler Barry, 2000), las instituciones de educación superior públicas y privadas se han visto en la obligación de tomar medidas innovadoras en aras de ofrecer una educación de calidad, que permita formar de manera competitiva la diversidad del individuo, con miras a las exigencias y necesidades del creciente mercado laboral. Es importante entonces que las instituciones educativas realicen cambios importantes en su proceso formativo, considerando la posibilidad de un modelo educativo constructivo y significativo que se ajuste permanentemente para alcanzar el desarrollo integral de todos sus estudiantes, partiendo de sus características individuales y posibilitando la generación de nuevas sociedades.

A partir de la observación y el trabajo insitu durante dos años en la institución donde se desarrolló la investigación, entidad que abandera procesos de inclusión de personas sordas, se ha identificado que las estrategias implementadas en aula no han garantizado en su totalidad la calidad y equidad educativa, la permanencia y promoción de este grupo poblacional. Razón por la cual, se propone en primera instancia establecer las necesidades educativas de los estudiantes sordos y a su vez, determinar las estrategias de enseñanza empleadas por los docentes en el aula con los estudiantes sordos, evidenciando la concordancia entre el horizonte institucional, la planeación curricular y la práctica docente, y tras determinar estos dos aspectos, conduce a proponer una intervención educativa orientada al diseño de estrategias de enseñanza que respondan a las necesidades, habilidades y capacidades de la comunidad, posibilitando la toma de decisiones en beneficio principalmente de los estudiantes sordos y apuntando al mejoramiento de la calidad educativa. 


\section{Estrategias de enseñanza aprendizaje en el proceso formativo de estudiantes sordos en la educación superior}

Por otro lado, la investigación abre la posibilidad que otras de las instituciones de educación superior que encabezan procesos de inclusión a nivel local, se vean incentivadas a generar planes de mejoramiento en sus prácticas pedagógicas partiendo de la comprensión de un diseño universal de aprendizaje, que responderá a las estrategias de enseñanza-aprendizaje de toda su comunidad educativa, para garantizar un real ejercicio de educación inclusiva.

En este contexto, el marco referencial que sustenta el estudio, está relacionado con las concepciones adoptadas sobre los conceptos de educación inclusiva en educación superior y educación inclusiva de personas sordas en la vida universitaria, tomando como referencia principal el modelo socio antropológico de la sordera que plantea Veinberg (2007) y los los Lineamientos de Política de Educación Superior Inclusiva del Ministerio de Educación Nacional (2013), por otro lado, se aborda el concepto de diseño universal de aprendizaje aplicado en la educación superior, retomado principalmente por Díez Villoria \& Sánchez Fuentes (2014), del mismo modo se recopila información sobre el enfoque socio cognitivo de aprendizaje que plantea Monereo (2007), analizando las estrategias de aprendizaje que plantea luego de diferentes investigaciones.

\section{Educación Inclusiva}

En los últimos años, la educación inclusiva se ha convertido en un tema de interés y de debate a nivel mundial, donde particularmente en el marco de las políticas públicas, se observa la necesidad de ajustar y adaptar la educación a grupos que han sido excluidos a lo largo de la historia, transitando más allá de las prácticas asociadas a la exclusión y discriminación y adentrándose sobre un real proceso de educación inclusiva.

De acuerdo con la Declaración mundial sobre Educación Superior (Unesco, 1998) las instituciones de educación, y en especial las universitarias, son las convocadas a asumir el compromiso social en la superación de tales prácticas, además de la generación de conocimiento, la garantía y el disfrute de la educación como un derecho humano fundamental y un bien público. Para la UNESCO este proceso es indispensable para alcanzar y proteger otros derechos humanos fundamentales; su reconocimiento y disfrute, reducen las desigualdades en las oportunidades y la disponibilidad de los recursos en una sociedad (Unesco, 2009).

En este orden de ideas, es preciso resaltar lo establecido desde diferentes Lineamientos Internacionales: en un primer lugar, la Convención sobre los Derechos de las Personas con Discapacidad señala, que los valores que promueve el paradigma de la inclusión se basan en el reconocimiento de las personas con discapacidad como sujetos de derecho y que todas las personas con discapacidad, como sujetos políticos, tienen derecho no sólo a manifestar sus necesidades y deseos, sino también a participar con sus opiniones en la toma de decisiones sobre todos aquellos aspectos que los afectan o inciden sobre su vida personal y el ejercicio de su ciudadanía (Fajardo, 2017).

En segundo lugar, el Informe Mundial sobre la Discapacidad del 2011, propone medidas para todas las partes interesadas - incluidos los gobiernos, las organizaciones de la sociedad civil y las organizaciones de personas con discapacidad - para crear entornos favorables, promover la rehabilitación y los servicios de apoyo, asegurar una adecuada protección social, crear políticas y programas inclusivos, y aplicar normas y legislaciones, nuevas o existentes, en beneficio de las personas con discapacidad y la comunidad en general (OMS- Organización Mundial de la salud, 2011).
Finalmente, el Observatorio Latinoamericano de Políticas Educativas, apoyado por la Fundación Ford con el auspicio de la Secretaría Especial de Promoción de Políticas de Igualdad Racial del Gobierno Federal de Brasil (Sverdlick, Ferrari, \& Jaimovich, 2005) afirma, tras la realización de una investigación, que es posible distinguir dos grandes grupos de políticas inclusivas en Latinoamérica: dentro del primer grupo, políticas mayoritarias que están representadas, en becas de ayuda económica, las cuales combinan como requisitos de otorgamiento un buen rendimiento académico sumado a la necesidad económica y en un segundo grupo las políticas centradas en la atención a la diversidad, evidenciadas en países como Brasil, Colombia o México (Fajardo, 2017).

En consecuencia y de acuerdo con el propósito del presente estudio, se tiene en cuenta que la educación inclusiva en Colombia es un concepto relativamente nuevo desarrollado a partir de diferentes enfoques que van más allá de la integración y el acceso al sistema, de acuerdo con los Lineamientos de la Política de Educación superior Inclusiva (Ministerio de Educación Nacional - MEN, 2013), la educación inclusiva es una estrategia central para luchar contra la exclusión social, que hace parte de una combinación de alternativas que buscan promover e implementar el proceso aferente a la inclusión social; en conclusión, de acuerdo con Opertti (2008) es un "principio rector general" que busca potenciar y valorar la diversidad, entendiéndola y protegiéndola, lo cual implica garantizar, según el contexto colombiano, las identidades y particularidades de los estudiantes, promover el respeto a ser diferente y facilitar la participación de la comunidad dentro de una estructura intercultural favoreciendo así la cohesión social, que es una de las finalidades de la educación (Blanco Guijarro, 2008).

\section{La comunidad sorda en la educación superior}

Veinberg (2007) define una concepción socio antropológica de la sordera, la cual se fundamenta en el hecho que los sordos son una minoría lingüística comparable a otros niños hablantes no nativos del español, con la diferencia que la modalidad de recibir y transmitir su lengua es viso-gestual en lugar de auditivo-oral. A partir de esta premisa, comienzan a evidenciarse representaciones sociales en donde el sordo es un ser sociolingüístico diferente que requiere unas alternativas pedagógicas distintas y que usa como lengua materna, la lengua viso espacial, reconocida como la lengua de señas.

Esta nueva concepción de la comunidad sorda y la aceptación de la lengua de señas en el campo educativo, aumenta las expectativas de la población y busca que las instituciones educativas ofrezcan un ambiente que supla las necesidades comunicativas y desarrolle las potencialidades cognitivas, sociales, culturales y lingüísticas, generando así condiciones bilingües-biculturales.

\begin{abstract}
La educación bilingüe bicultural para sordos, EBBS, posibilita el cumplimiento de este derecho al ser una propuesta educativa que responde coherentemente a la situación, condiciones y características históricas, sociolingüísticas y escolares de la comunidad sorda del país. Para ello, la EBBS propicia transformaciones profundas en los procesos ideológicos, políticos, educativos y comunitarios que expresan la sociedad y sus instituciones, sobre las personas sordas y la sordera (INSOR, 2006, p. 11).
\end{abstract}

A partir de la nueva perspectiva, cambia por completo la forma de abordar la educación de sordos, porque comprende las particularidades de la población, teniendo en cuenta sus propias necesidades y 
reconociendo su lengua. Al ser la lengua de señas, su lengua materna o primaria, la comunicación es entendida desde el modelo Sistémico de la Comunicación interpersonal como un fenómeno social de interacción cultural y como una esfera constituyente de lo humano, una de las determinantes del bienestar del hombre y por ende de su calidad de vida. Se asume al hombre como un ser cultural y social por excelencia, y que se humaniza a través de la comunicación; significa eso que la existencia del hombre sólo es posible a través de la comunicación y la de ésta, a través de la existencia del hombre. Por lo tanto, sin comunicación es imposible concebir a la sociedad y al hombre mismo (Bernal, Pereira Alba, \& Rodríguez Jiménez, 2018).

Pensar en implementar un modelo bilingüe bicultural en la educación superior para sordos, supone visiones desde los sordos y desde los oyentes, y trasciende a la totalidad del sistema educativo y su reestructuración de condiciones y vida institucional.

\section{Massone, Druetta y Simon (2003, p. 67) refieren que pensar en una verdadera transformación educativa para el caso de los sordos no implica la mera inclusión de la lengua de señas en las instituciones educativas, como se interpreta a partir de muchos de los discursos de sordos y de profesio- nales oyentes del país y del exterior que postulan el bilin- güismo para los sordos, (Morales García, 2015).}

Teniendo en cuenta estas características, es importante abordar las implicaciones de la implementación de un modelo pedagógico con unos lineamientos curriculares y estrategias educativas que permitan organizar y evaluar los procesos educativos de las personas sordas, garantizando el cumplimiento de los objetivos planteados caracterizados por su diversidad lingüística y cultural. Ante las dificultades observadas desde lo académico y comunicativo de los estudiantes sordos en la educación superior, se propone enfatizar en las estrategias que se deberían implementar en aula para facilitar el acceso a la información y conceptualización académica.

\section{Diseño Universal de Aprendizaje}

Definido por el Dr. David Rose, cofundador del Center for Applied Special Technology (CAST) como aquella respuesta dirigida a todos los aprendices, en el cual se busca eliminar todas las barreras para aprender, busca crear currículos que atiendan las necesidades de todos los estudiantes desde el primer momento, se trata de un sistema de apoyo que favorece la eliminación de barreras físicas, sensoriales, afectivas y cognitivas para el acceso, aprendizaje y la participación de los alumnos (Giné Giné \& Font, 2007).

\section{El Diseño Universal para el Aprendizaje (DUA) es un marco que aborda el principal obstáculo para promover aprendi- ces expertos en los entornos de enseñanza: los currículos inflexibles, "talla-única-para-todos". Son precisamente estos currículos inflexibles los que generan barreras no intencionadas para acceder al aprendizaje. Los estudian- tes que están en "los extremos", como los superdotados y con altas capacidades o los alumnos con discapacidades, son particularmente vulnerables. Sin embargo, incluso los alumnos que se pueden considerar "promedio" podrían no tener atendidas sus necesidades de aprendizaje debido a un diseño curricular pobre (Pastor, Sánchez Hípola, Sánchez Serrano, \& Zubillaga del Río, 2013).}

La aplicación del DUA a la educación superior se reconoce como el enfoque del diseño instruccional universal (DIU), que, en resumen, plantea la aplicación de los siete principios del diseño universal arquitectónico al ámbito pedagógico. Se define, como el incremento de las capacidades instruccionales en entornos universitarios, a través de la potenciación y el fomento de las competencias y los conocimientos del profesorado, y bajo esta línea se podría hablar de la inmersión del aprendizaje estratégico a la vida universitaria (CAST -Center for Applied Special Technology-, 2013).

El Diseño Universal aplicado a la enseñanza universitaria - DUI es un proceso a través del cual se pueden identificar y eliminar las barreras en la enseñanza y el aprendizaje, potenciando al mismo tiempo el aprendizaje de todos los estudiantes y reduciendo la necesidad de realizar adaptaciones específicas; requiere de planificación, diligencia y flexibilidad. El DUI se convierte en un recurso de apoyo al docente universitario en su práctica y es aplicable a cualquier rama de estudios, asignatura, grado de experiencia del profesorado y el nivel de estudios (grado, posgrado, máster y doctorado) (Dalmau Montalá, y otros, 2002).

No obstante, el docente universitario, debe recibir formación y tener un acompañamiento institucional a la hora de proporcionar las mismas oportunidades para todos los estudiantes, independientemente de sus características individuales, respondiendo a estándares de calidad en la educación superior y reconociendo la diversidad en el aula.

\section{Aprendizaje estratégico}

De acuerdo con las nuevas exigencias contextuales y los cambios que se viven en esta sociedad, caracterizada por la globalización e influenciada de desarrollos tecnológicos, es importante que las instituciones educativas promuevan el desarrollo de aprender a aprender a lo largo de la vida y que las personas adquieran diferentes y nuevas habilidades para el aprendizaje.

Uno de los propósitos más importantes del acto educativo en la historia, ha sido el de enseñar a los estudiantes a ser personas independientes, autónomas y con poder de decisión, a través de la mediación implícita del aprendizaje de diversos dominios de tipo cognitivo, social y conductual, los cuales le permitan responder a las demandas de la sociedad. Marín Ortega \& Moreno Hernández (2007) definen "aprender a aprender" como la competencia que le permite a la persona dominar las capacidades para el aprendizaje y la reflexión en cómo se aprende, más conocida como metacognición, a partir del autoconocimiento y de la construcción de un sentimiento de eficacia.

En este sentido, las instituciones de educación superior están comprometidas en realizar una renovación curricular para que se cumpla con este propósito, pues es en la educación superior donde se produce con mayor presteza la expiración de los conocimientos; la formación profesional es un proceso autónomo y de autodeterminación, en donde se han adquirido habilidades sociales que permitirían el desarrollo del aprendizaje estratégico.

Para Monereo (2000) el proceso metacognitivo o el "aprender estratégico" embarca procesos de toma de decisiones, por lo tanto intencionales, que el estudiante selecciona para enfrentarse a los conocimientos que necesita para cumplimentar una determinada demanda u objetivo, dependiendo de las características de la situación educativa, de igual manera señala que debe darse desde tres puntos emergentes: desde la persona, partiendo de sus capacidades, de lo que sabe y de las personas que se relacionará mientras aprende, desde la tarea, partiendo de las estrategias a implementar y las dificultades propias de las actividad y desde el contexto, teniendo en cuenta variables como: características del entorno, acto comunicativo y limitaciones vs barreras. 


\section{Estrategias de enseñanza aprendizaje en el proceso formativo de estudiantes sordos en la educación superior}

Por lo tanto, hay que preguntarse cómo se está llevando el proceso de aprender a aprender en las aulas diversas de hoy en día, reflexionar si se dispone de estrategias de aprendizaje pertinentes que respondan a las características individuales de los estudiantes, lo cual implica, que el docente sepa cuál, cómo, cuándo, dónde y por qué aplicar dichas estrategias y de igual manera, cada institución educativa debe disponer bajo qué formato organizativo serán enseñadas las estrategias de aprendizaje. La posibilidad del estudiante de aprender a aprender resulta cada vez más consciente de su proceso de cognición, es decir, de la metacognición, proceso que se refiere al conocimiento o conciencia que tiene la persona de sus propios procesos mentales, sobre cómo aprende y al control del dominio cognitivo, sobre su forma de aprender. Ambos se orientan al servicio de una mejora del estudio personal que les conduzca a resultados satisfactorios de aprendizaje (Monereo, 2007).

Un aprendizaje estratégico busca entre los estudiantes conservar la capacidad de asombro, ser persistente, plantearse preguntas, disminuir la impulsividad, transferir conocimientos, escuchar la opinión de otros, usar lenguaje con precisión, ser creativo, tener flexibilidad de pensamiento, usar todos los sentidos, chequear la adecuabilidad de las respuestas y ejercitar la metacognición. Partiendo del conocimiento de sí mismo, específicamente con el dominio que se tiene sobre la actividad intelectual propia, y que es una expresión de pensamientos de orden superior que hacen posible la autoconciencia, regulación y control de la actividad cognitiva propia cuando se está llevando a cabo una tarea intelectualmente exigente (González, 2009).

\section{Estrategias de aprendizaje}

Son procesos de toma de decisiones, por lo tanto, intencionales, que el alumno selecciona para enfrentarse a los conocimientos que necesita para cumplimentar una determinada demanda u objetivo. Desde el paradigma socio - cognitivo, por una parte, se reconoce la importancia de la construcción personal de representaciones mentales de la realidad y su función mediadora en los procesos cognitivos de gestión de la información y, por otra, legitima que, en esa construcción, los materiales y herramientas empleados tienen un origen, un desarrollo y un sentido social (Monereo, 2007). Por consiguiente, el acto educativo debe propender por formar individuos autónomos, independientes y autorregulados, a través de un contexto estratégico que permee las concepciones de los estudiantes y docentes, frente al sentido y significado de aprender y enseñar.

Román y Gallego (1994) citado por Pizano Chavez (2014) proponen una clasificación de estas estrategias, organizadas así: estrategias de adquisición de información, estrategias de codificación de información, estrategias de recuperación de información y estrategias de apoyo al procesamiento de la información.

Tabla 1. Clasificación de las estrategias de aprendizaje.

\begin{tabular}{|c|c|c|c|}
\hline $\begin{array}{l}\text { Estrategias de } \\
\text { adquisición de } \\
\text { información }\end{array}$ & $\begin{array}{l}\text { Estrategias de } \\
\text { codificación de } \\
\text { información }\end{array}$ & $\begin{array}{l}\text { Estrategias de } \\
\text { recuperación de } \\
\text { información }\end{array}$ & $\begin{array}{l}\text { Estrategias de } \\
\text { apoyo al } \\
\text { procesamiento de } \\
\text { la información }\end{array}$ \\
\hline $\begin{array}{l}\text { Estrategias } \\
\text { atencionales }\end{array}$ & $\begin{array}{c}\text { Estrategias de } \\
\text { nemotecnización }\end{array}$ & $\begin{array}{l}\text { Estrategias de } \\
\text { búsqueda }\end{array}$ & $\begin{array}{c}\text { Estrategias } \\
\text { metacognitivas }\end{array}$ \\
\hline $\begin{array}{l}\text { Estrategias de } \\
\text { repetición }\end{array}$ & $\begin{array}{l}\text { Estrategias de } \\
\text { elaboración }\end{array}$ & $\begin{array}{l}\text { Estrategias de } \\
\text { generación de } \\
\text { respuesta }\end{array}$ & $\begin{array}{c}\text { Estrategias } \\
\text { afectivas }\end{array}$ \\
\hline & $\begin{array}{l}\text { Estrategias de } \\
\text { organización }\end{array}$ & & Estrategias sociales \\
\hline
\end{tabular}

\section{Estrategias de enseñanza}

Para hablar de estrategias de enseñanza es necesario reconstruir el concepto de aprendizaje; gracias a la literatura podemos encontrar tres visiones diferentes pero interrelacionadas: psicológica, disciplinaria y socio histórica.

\begin{abstract}
La visión psicológica del aprendizaje es desde la cual se hacen consideraciones acerca del funcionamiento mental del ser humano (conductista vs cognoscitivista); la disciplinaria, en este caso examina los procesos y productos de las diferentes disciplinas escolares; y la socia histórica, donde lo que se toma en cuenta son las situaciones sociales en las que se produce el aprendizaje (contexto, teoría de la actividad, prácticas sociales) (González, 2009, p. 10).
\end{abstract}

En este estudio, se tiene en cuenta las tres visiones, teniendo en cuenta que nos focalizamos en el acto de aprender a aprender, Weinstein (1986) citado por Monereo (2007), refiere que el aprender a aprender no se refiere al aprendizaje directo de contenidos, sino al aprendizaje de habilidades con las cuales aprender contenidos. De esta manera, aprender a cómo aprender es un ejercicio de evaluación y reflexión de la práctica docente y el de disponer e innovar en el uso de estrategias de aprendizaje, de acuerdo con González (2009), el docente debe saber cuál, cómo, cuándo, dónde y por qué aplicar estas estrategias.

La enseñanza de estrategias de aprendizaje, desde el enfoque histórico cultural, se centra en la interrelación enseñanza y desarrollo personológico, como expresan Díaz Barriga \& Hernández Rojas (2007), de ahí que no se limita únicamente a la adquisición de estrategias de aprendizaje y su uso por parte de los estudiantes, pues requiere de otros procedimientos de los cuales el docente debe estar consiente, Rué (2010, p. 23) establece que "el docente requiere de la activación de los conocimientos conceptual o declarativo, procedimental, y estratégico o condicional; así como de los momentos reguladores en la acción de aprendizaje, en su preparación, en su desarrollo y en sus resultados".

\section{Metodología}

Esta investigación en términos metodológicos se propone a partir de la investigación cualitativa interpretativa que, de acuerdo con Hernández Sampieri, Fernández Collado, \& Baptista Lucio (2014, p. 9) "se fundamenta en una perspectiva interpretativa centrada en el entendimiento del significado de las acciones de seres vivos, sobre todo de los humanos y sus instituciones (busca interpretar lo que va captando actuvamente)". El método empleado es investigación acción, que como señala Sandín (2003) citado por Hernández Sampieri, Fernández Collado, \& Baptista Lucio (2014, p. 496) “(...) pretende, esencialmente, propiciar el cambio social, transformar la realidad (social, educativa, económica, administrativa, etc.) y que las personas tomen conciencia de su papel en ese proceso de transformación".

La muestra poblacional estuvo conformada de la siguiente manera:

$\rightarrow$ Cuatro (4) estudiantes sordos matriculados oficialmente al programa de Lic. en Educación Especial en el semestre $2^{\circ}, 5^{\circ}$ y $6^{\circ}$, los cuales corresponden al 50\% de la población sorda total matriculada en la licenciatura en modalidad presencial. Los estudiantes tienen entre 20 a 30 años, son usuarios de la Lengua de Señas Colombiana (LSC) y han recibido su proceso de formación en la modalidad de modelo lingüístico. 
$\Rightarrow$ Cuatro (4) docentes de la facultad, que imparten diferentes asignaturas a los estudiantes.

$\rightarrow \quad \mathrm{Y}$ dos (2) intérpretes asignados a las clases de los docentes seleccionados.

\section{Técnicas e instrumentos}

Inicialmete se realizó una inmersión total a las aulas, con autorización de la institución y de los docentes, con el fin de reconocer el contexto y definir una muestra tentativa, luego de tener la muestra tentativa, se procedió a establecer la muestra inicial, recalcando que ésta no representa a la población, pero permite cumplir con el propósito del estudio.

Al establecer la muestra inicial, se programaron las sesiones de observación participante que según Taylor \& Bogdan (1987) involucra la interacción social entre el investigador y los informantes en el escenario social, ambiente o contexto de los últimos, y durante la cual se recogen datos de modo sistemático y no intrusivo.

Durante el ejercicio de observación se implementaron guías de observación y notas de campo, al igual que toma de fotografías y video grabaciones, como instrumento de recolección de la información.

La observación estructurada no participante se realizó inicialmente para explorar y describir el contexto del aula, la relación que se da entre el docente y el estudiante, el estudiante y el intérprete y el docente y el intérprete. El formato utilizado para este primer paso fue una Guía de Observación y como insumo de recolección de la información un formato de nota de campo, siguiendo las indicaciones de anotación referidas por Hernández Sampieri, Fernández Collado, \& Baptista Lucio (2014). A partir de aquí se seleccionaron los docentes a observar y las clases en las cuales tienen estudiantes sordos.

En un segundo momento, se planearon tres sesiones de observación estructurada no participante y tres de observación participante, en las asignaturas dadas por los docentes seleccionados en la muestra, en las cuales estaban todos los estudiantes sordos de la muestra escogida, es de aclarar que estas intervenciones estaban programadas con el fin de establecer las estrategias de aprendizaje utilizadas por los estudiantes sordos dentro del aula y no observar al docente; para realizar este proceso se diseñó una Guía de Observación parametrizada según la clasificación de las estrategias de aprendizaje que nos refiere Román \& Gallego (1994) y validada por dos docentes expertos; las sesiones de observación participante coincidían con encuentros programados interclases desde la planeación curricular. Como insumo de recolección de la información se utilizó el formato de nota de campo.

Para el tercer momento de la observación, se programaron dos sesiones por docente en las cuales, a partir de una observación no participante, se establecerían en un primer momento, las estrategias de enseñanza implementadas por los docentes con los estudiantes sordos. Como instrumento de recolección se hizo uso de la Guía de Pauta de Observación a Docentes, establecida por el Ministerio de Educación Nacional (2008), la cual hace parte de la Guía Metodológica de evaluación Anual a docentes y directivos del Ministerio de Educación Nacional- MEN.

Para este proceso no se hicieron ajustes metodológicos de ninguna índole y con el fin de obtener una observación acertada que tuviese presente la integralidad, se organizaron las categorías y subcategorías en relación con los objetivos de la investigación.
Luego de realizar las sesiones de observación, se procede a realizar entrevistas semiestructuradas; para las entrevistas a los estudiantes se diseñó un instrumento con siete (7) preguntas, el cual fue validado por dos docentes expertos en investigaciones con población sorda, el fin de la entrevista con los estudiantes fue obtener información sobre las condiciones que resultan más favorables en su proceso de aprendizaje y establecer que estrategias de aprendizaje usan de acuerdo a la clasificación de Román Sánchez \& Gallego Rico (2008), teniendo en cuenta que la muestra seleccionada es usuaria de lengua de señas, se requirió contar con el servicio de interpretación en cada una de las entrevistas; de conformidad con el numeral 25 del artículo $1^{\circ}$ de la Ley 982 de 2005 los intérpretes para sordos son personas con amplios conocimientos de la Lengua de Señas Colombiana que puede realizar interpretación simultánea del español hablado en la Lengua de Señas y viceversa (Congreso de Colombia, 2005).

Como se mencionó anteriormente, se hicieron ajustes metodológicos tales como obtener la información a través de video grabación y garantizando el servicio de interpretación a la población Sorda de tal manera que se sintieran cómodos en el momento de la entrevista y se hizo transcripción fiel de sus intervenciones a español escrito. Sin embargo, es importante reconocer que la relación existente con los estudiantes sordos y el conocimiento de lengua de señas permite identificar y analizar las variables más efectivamente.

De otro lado se diseñaron los cuestionarios para la aplicación de entrevistas a los cuatro docentes y dos intérpretes, los instrumentos fueron validados por docentes expertos. Para las entrevistas se tomó en cuenta la disponibilidad de los participantes, se hicieron de manera individualizada, se les entregó un consentimiento informado y fueron grabadas en audio, facilitando la transcripción para el análisis.

Para la entrevista a los docentes se diseñaron ocho (8) preguntas y para los intérpretes seis (6) preguntas, organizadas en cuatro (4) categorías: identificación, trayectoria, rol y estrategias de enseñanza; la entrevista de los docentes tiene el propósito de identificar las estrategias que utilizan en sus clases específicamente con los estudiantes sordos, y la entrevista de los intérpretes permite identificar el rol que desempeña dentro del proceso de enseñanza aprendizaje, de igual manera se realizaron dos grupos focales, para los cuales, se diseñaron los cuestionarios que fueron validados por docentes expertos.

Por otro lado, como recurso organizativo, con el fin de facilitar y agilizar la tabulación, hacer un análisis meticuloso, confiable y válido, para el respaldo en las conclusiones, y facilitar la realización del informe final, se codificaron todos los insumos de recolección de datos, partiendo de la codificación general de los instrumentos utilizados.

Para el análisis de datos se empleó Atlas ti. (8.0), el cual compone una herramienta de mucha utilidad para el almacenamiento, sistematización y análisis de la información, basado en la Teoría Fundamental propuesta por los sociólogos Glaser y Strauss en la década de los años sesenta, sustentada a su vez en la teoría del interaccionismo simbólico de Blúmer y las ideas del pragmatismo de la escuela de Chicago de Mead y Dewey, según como lo proponen Taylor \& Bogdan (1987) citado por Varguillas (2006). En el programa, el proceso implica cuatro etapas: codificación de la información, categorización; estructuración o creación de una o más redes de relaciones entre las categorías; y estructuración de hallazgos o teorización si fuere el caso.

\footnotetext{
Atlas ti. es un paquete de software especializado en análisis cualitativo de datos que permite extraer, categorizar e inter-vincular segmentos de datos desde diversos documen-
} 
tos. Basándose en su análisis, el software ayuda a descubrir patrones. La unidad hermenéutica es la estructura que contiene los datos y operaciones que realizamos. Contiene: documentos primarios, quotations, codes, memos, familias, y networks" (Sabariego Puig, Vila, \& Sandín Esteban, 2014, p. 123).

Para lograr la fase de categorización, se prepararon los documentos tal y como los requiere el software, se organizaron las guías de observación, se clasificaron las notas de campo, se transcribieron las entrevistas y los grupos focales, al igual que los videos tomados en la observación.

Con apoyo de la herramienta se logra realizar las tres etapas de análisis: a) análisis teórico, b) análisis metodológico, y c) análisis final a través de una triangulación de datos, dando confiabilidad y validez a los resultados, como derivación de la sistematización para el análisis e interpretación de los datos se instituyeron las categorías o unidades de análisis, teniendo en cuenta los temas principales planteados en la investigación: estrategias de aprendizaje en estudiantes sordos (EAS), estrategias de enseñanza implementadas por los docentes para los estudiantes sordos (EED) y relación del proceso de enseñanza - aprendizaje en estudiantes sordos (REA).

\section{Resultados}

A continuación, se presentan los resultados obtenidos tras el proceso de tratamiento e interpretación de los datos, el cual suscita de acuerdo con los objetivos de la investigación.

En primer lugar, se describen los resultados obtenidos frente a las estrategias de aprendizaje que utilizan los estudiantes sordos dentro del aula inclusiva, posteriormente se describen los resultados en relación con las estrategias de aprendizaje que se observan y refieren los docentes y luego se describen las concepciones evidenciadas frente al proceso de enseñanza aprendizaje de los estudiantes sordos en un aula inclusiva.

Es justo recordar que la importancia de la presente investigación reside en la revisión, análisis, reflexión y contrastación de los instrumentos aplicados para la recolección de la información. El procedimiento se basó inicialmente en la lectura sistémica y profunda de cada una de las notas de campo, de las entrevistas y de los grupos focales, al igual que la visualización detallada de los videos y su respectiva transcripción, destacando los elementos discursivos allí presentes que hacían alusión a las unidades de análisis establecidas.

En la siguiente tabla, se definen las subcategorías de acuerdo con la encontrado en la aplicación de los instrumentos y de acuerdo con las unidades de análisis:

Tabla 2. Definición de subcategorías

\begin{tabular}{|c|c|c|}
\hline CÓDIGO UNIDAD DE ANÁLISIS & SUBCATEGORÍA & DEFINICIÓN \\
\hline \multirow{4}{*}{ EAS } & $\begin{array}{l}\text { Estrategias de } \\
\text { adquisición de } \\
\text { información }\end{array}$ & $\begin{array}{l}\text { Procesos cognitivos básicos que emplean los estudiantes sordos para captar la } \\
\text { información en el aula de clase inclusiva. Respondiendo a preguntas como ¿cuál es el } \\
\text { proceso atencional del estudiante?,¿cómo centra su atención?, ¿en dónde centra la } \\
\text { atención el estudiante, en el intérprete o en el docente? }\end{array}$ \\
\hline & $\begin{array}{l}\text { Estrategias de } \\
\text { codificación de } \\
\text { información }\end{array}$ & $\begin{array}{l}\text { Procesos cognitivos básicos que emplean los estudiantes sordos en el aula, para } \\
\text { transformar, organizar, estructurar y comprender la información recibida en el aula, } \\
\text { teniendo en cuenta que ésta no se recibe directamente del docente, sino con el mediador } \\
\text { comunicativo o intérprete. }\end{array}$ \\
\hline & $\begin{array}{l}\text { Estrategias de } \\
\text { recuperación de } \\
\text { información }\end{array}$ & $\begin{array}{l}\text { Procesos cognitivos básicos que emplean los estudiantes sordos al salir del aula para } \\
\text { recuperar la información obtenida en cada una de las clases. Respondiendo a preguntas } \\
\text { como ¿qué sistemas de búsqueda o generación de respuestas manejan los estudiantes? }\end{array}$ \\
\hline & $\begin{array}{l}\text { Estrategias de } \\
\text { apoyo de } \\
\text { información }\end{array}$ & $\begin{array}{l}\text { Procesos metacognitivos empelados por los estudiantes sordos para optimizar los } \\
\text { anteriores procesos, apoyos extracurriculares que emplean y motivacionales en el proceso } \\
\text { de aprendizaje. }\end{array}$ \\
\hline \multirow{4}{*}{ EED } & $\begin{array}{l}\text { Estrategias } \\
\text { visuales }\end{array}$ & $\begin{array}{l}\text { Acciones coordinadas o dirigidas por los docentes para dar a conocer el tema de clase, } \\
\text { representados en apoyos visuales como videos, imágenes, mapas metales, mapas } \\
\text { conceptuales entre otros. }\end{array}$ \\
\hline & $\begin{array}{l}\text { Estrategias } \\
\text { auditivas } \\
\text { ajustadas }\end{array}$ & $\begin{array}{l}\text { Acciones coordinadas o dirigidas por los docentes para dar a conocer el tema de clase, } \\
\text { representados en apoyos visuales como videos con subtitulación, closed caption, o } \\
\text { interpretación en lengua de señas. }\end{array}$ \\
\hline & $\begin{array}{l}\text { Estrategias } \\
\text { sociales }\end{array}$ & $\begin{array}{l}\text { Acciones coordinadas o dirigidas por los docentes para lograr interacción entre } \\
\text { estudiantes sordos y oyentes dentro del aula de clase. }\end{array}$ \\
\hline & $\begin{array}{l}\text { Estrategias de } \\
\text { lectura }\end{array}$ & $\begin{array}{l}\text { Acciones coordinadas o dirigidas por los docentes para fortalecer y promover la lectura en } \\
\text { español con los estudiantes sordos. }\end{array}$ \\
\hline \multirow{2}{*}{ REA } & $\begin{array}{l}\text { Mediador } \\
\text { comunicativo }\end{array}$ & $\begin{array}{l}\text { Mediación comunicativa en las clases a partir del servicio de interpretación dentro y fuera } \\
\text { del aula. Acción que se desarrolla tanto con docentes como con pares académicos. }\end{array}$ \\
\hline & $\begin{array}{l}\text { Ajustes en la } \\
\text { evaluación }\end{array}$ & $\begin{array}{l}\text { Acciones realizadas por el docente para evaluar a los estudiantes sordos en su primera } \\
\text { lengua: lengua de señas colombiana. }\end{array}$ \\
\hline
\end{tabular}




\section{Estrategias de aprendizaje en estudiantes sordos (EAS).}

A través de la observación, la entrevista y el grupo focal, se encontró que, en los estudiantes sordos seleccionados como muestra, existen características individuales y generales en su proceso de enseñanza aprendizaje, todo ello dependiendo del momento en el que adquirieron la lengua de señas y del proceso formativo de educación prescolar y básica primaria.

Entre los elementos que surgen al establecer lo que refieren los estudiantes, la percepción que tienen frente a su proceso formativo y lo que se vive en el aula día a día, se revela de entrada en términos generales, la importancia del acto comunicativo y la mediación que se establece a partir del servicio de interpretación, tomando protagonismo el rol que desempeña el intérprete en el proceso de enseñanza aprendizaje.

Tal y como lo afirma Vygotsky (1995a) citado por Castilla, Ayala Cardona, \& Acosta Pineda (2015) es importante precisar que la intervención del lenguaje juega un papel central en el desarrollo del autocontrol, la orientación en las tareas, la resolución de problemas y en el desarrollo social del sujeto, en la medida en que permite organizar nuestro pensamiento, categorizar el mundo por medio de conceptos y mediar con su cultura. Al ser el intérprete la persona con la que puede entablar una comunicación fluida, este se convierte en la herramienta principal para el estudiante en su proceso formativo.

Por otro lado, aunque cada uno de los estudiantes tiene un ritmo de aprendizaje diferente, se encontró que:

$\rightarrow$ Frente a las estrategias de adquisición de información centran su atención en la información que les está dando el intérprete, obviando en algunas ocasiones las situaciones que se están presentando en el contexto, como gestos del docente, articulación con la presentación, entre otros, asumiendo en su totalidad la información dada por el intérprete.

$\rightarrow$ Frente a las estrategias de codificación de información recopilan la información dada por el mediador comunicativo que interpreta en lengua de señas lo hablado u orientado por el docente. No hay una toma de notas sistemática de la clase, sino un proceso de memorización de lo que más puedan retomar de la clase y de algunos apuntes de sus compañeros o de la presentación dada por el docente en medio magnético.

$\rightarrow$ Frente a las estrategias de recuperación de información muestran habilidades en la memoria a corto, mediano y largo plazo en la información dada en las diferentes asignaturas, dependiendo del interés, gusto y motivación que tengan de la misma. Por otro lado, acuden a tutorías con el docente o repetitivamente a preguntar directamente al intérprete. Estando dentro del aula, si tienen dudas sobre el tema de la clase, prefieren preguntar al interprete primero y si el intérprete no da la respuesta que ellos esperan preguntan al docente.

$\Rightarrow$ Finalmente, como estrategias de apoyo de información no hacen uso de ninguna técnica de estudio, toman fotografía de las presentaciones o de los cuadernos de sus compañeros y si la clase es de su interés y tienen una buena relación con el docente, le solicita tutoría particular o recursos bibliográficos extras.

Se presentó una tendencia en los estudiantes en los momentos de observación no participante y fue el uso de señas sin dirección a ningún receptor, lo que es conocido como lenguaje egocéntrico, evi- denciando que lo realizaban para comprender y confirmarse a sí mismo la información que le estaba llegando a través del intérprete o desde la presentación visual dada por el docente. Esta situación permite interpretar la importancia de la comunicación en cualquier situación, como se nombraba anteriormente el desarrollo del lenguaje va ligado al desarrollo de pensamiento.

\section{Estrategias de enseñanza implementadas por los docentes para los estudiantes sordos (EED).}

Se identifica que cada docente, según lo refieren, reconoce la importancia del rol que desempeña el intérprete al igual que la necesidad de conocer conceptos básicos de lengua de señas. Para interactuar directamente con los estudiantes proponen estrategias metodológicas que puedan ser de uso para todos en el aula, sin embargo, hacen hincapié en la necesidad de adaptar algunos materiales o ajustar algunos textos para que puedan ser de fácil acceso a todos los estudiantes.

Por otro lado, se detecta la concepción que al ubicar al estudiante sordo estratégicamente en el aula y el hacer que trabaje con sus compañeros sordos, desarrollará mejor las competencias dentro del aula, y además se motivará a interactuar con sus demás compañeros.

De igual manera, los docentes haciendo casi que una generalización, dan por concebido que el usar material visual, videos adaptados o estrategias gráficas permiten un mejor proceso de enseñanza aprendizaje en el aula y que es muy necesario hacer ajustes en los textos de lectura, para que ellos aprendan el español escrito.

Todo lo anterior permite categorizar las estrategias de enseñanza utilizadas por los docentes en: estrategias visuales, estrategias auditivas ajustadas, estrategias sociales y estrategias de lectura.

En este sentido se identifica con apoyo de los resultados de las anotaciones hechas bajo la Guía de Observación, que los docentes realizan un proceso de formación bajo el plan curricular establecido por la institución educativa, y aunque reconozcan y refieran que se deben realizar ajustes y/o adaptaciones, no tienen el conocimiento de cómo realizarlo con la población sorda.

\section{Relación del proceso de enseñanza - aprendizaje en estudiantes sordos (REA).}

Para esta categoría se tienen en cuenta los resultados de las observaciones, percepciones de los estudiantes, de los docentes y la percepción y opinión de los intérpretes en el proceso de formación de los estudiantes sordos. El intérprete es el mediador comunicativo de los estudiantes sordos y nace de la necesidad de integrarlos con estudiantes oyentes; en ese sentido la institución, actúa en la inclusión de estudiantes sordos, bajo un modelo integrador.

Al abordar la categoría de relación del proceso de enseñanza aprendizaje, el intérprete toma protagonismo al ser el mediador comunicativo, pero no se puede desconocer que no sólo cumplen esta función, porque también se vuelven pares de los docentes al momento de lograr transmitir la información tal cual el docente la está expresando 


\section{Estrategias de enseñanza aprendizaje en el proceso formativo de estudiantes sordos en la educación superior}

para todos los estudiantes, de igual manera al ser apoyo del docente en la generación de nuevas estrategias, por ser quienes conocen más de cerca la población, la cultura y al estudiante en particular. Por otro lado, son la fuente principal de información de los estudiantes, convirtiéndose de igual manera en un par académico para ellos.

\section{Discusión y Conclusiones}

El análisis de los resultados obtenidos en la aplicación de los instrumentos seleccionados y el análisis teórico que emerge del presente estudio permite dar respuesta a las preguntas de investigación y a su vez a los objetivos planteados.

De acuerdo con el primer objetivo y los resultados obtenidos en esta categoría, se puede establecer que los estudiantes sordos emplean diferentes estrategias no planificadas para facilitar su proceso de aprendizaje, están expuestos a aprender o no aprender de acuerdo con la didáctica que el docente maneje, no reconocen cuáles son sus habilidades y técnicas de estudio, ni como maximizar su proceso de aprendizaje, emplean estrategias de aprendizaje similares no consientes, a través de las cuales obtienen resultados diferentes dependiendo de la asignatura, el docente, el interés o motivación y el intérprete que acompañe la asignatura.

En algunos casos, sin saberlo ni haberlo identificado, es posible estar frente a personas sordas con algunas dificultades cognitivas debido a que la adquisición del lenguaje se da de manera tardía, y en muchas ocasiones la Lengua Oral (LO) es tomada como el ideal por parte de los padres, lo cual puede traer consecuencias negativas para el desarrollo del niño.

\section{Schorn (2008) citado por Fontal González \& Mejía Zulua- ga (2015) manifiesta que la falta de un lenguaje materno adecuado de forma temprana puede provocar consecuen- cias tales como conductas impulsivas, agresivas e incluso rasgos autísticos en el niño sordo, debido a que los padres se centran en la posibilidad de que su hijo sea oyente y pro- duzca el habla (Fontal González \& Mejía Zuluaga, 2015, p. 51).}

Por otro lado, los docentes de la licenciatura, a pesar de tener una vasta formación pedagógica, se están enfrentando a procesos de inclusión sin tener claridades teóricas, metodológicas y curriculares, al llegar al aula se enfrentan con un sinnúmero de estudiantes con características diversas y diferentes formas de aprendizaje, a los cuales no pueden dar respuesta; particularmente se enfrentan con los estudiantes sordos, con quienes no pueden mantener un diálogo directo y requieren de un tercero para poder comunicarse. Al no existir una estructura y un modelo a seguir, el estudiante sordo queda rezagado del proceso formativo al ser parte un grupo minoritario; es de aclarar que el docente sigue un plan curricular, un plan de curso y utiliza diversas estrategias metodológicas en el aula, desde su experiencia pedagógica, pero dichas estrategias van muy encaminadas al proceso formativo de estudiantes oyentes.

A razón de generar espacios educativos realmente inclusivos, es necesario plantear nuevas proyecciones y escenarios en donde el proceso de enseñanza - aprendizaje se dé, y más en la educación superior en donde se forman futuros profesionales que deben contar con unas competencias básicas para salir al mercado laboral.

A nivel general, de acuerdo con los resultados obtenidos, se definen las cuatro estrategias de aprendizaje como importantes, para facilitar el proceso aprendizaje y garantizar su éxito, de igual manera presentan una serie de técnicas que se integran entre sí. Según Monereo (2007), es necesario enseñar a los estudiantes a aprender a aprender, es decir, enseñar desde un aprendizaje estratégico.

Lo que se encuentra es que, como bien lo definen las estrategias de adquisición de la infomación, el primer paso para adquirir el conocimiento es atender, por eso con el fin de beneficiar los procesos de atención de los estudiantes, así como el control o dirección de todo proceso cognitivo hacia lo que en realidad es relevante para él, se recomienda: dar a conocer el plan de curso con anterioridad, facilitar el material de estudio antes de la clase, organizar el material y recurso de la clase, enseñarles a las estudiantes técnicas de lectura comprensiva, generar vocabulario y/o palabras claves en el proceso de la lectura, y discutir sobre los mismos en clase, todo esto desde su lengua materna.

Luego de que el estudiante adquiera el conocimiento es importante que codifique la información, tal y como lo plantean las estrategias de codificación de la información. Para ayudar a que la información no se pierda, es importante que se realicen las siguientes técnicas: reducir la información a una palabra clave o frase, construir imágenes, mapas, gráficos que representen la información recibida, construir mapas conceptuales o resúmenes ayuda a consolidar la información dada y captar lo más importante de ella para su aplicación.

Para fortalecer las estrategias de recuperación de la información y mejorar los sistemas de búsqueda en la memoria, es preciso que se tengan en cuenta las siguientes técnicas: tener la información ordenada, dar pautas y espacios a los estudiantes para que tomen apuntes.

En este sentido, el docente debe reconocer y responder a las estrategias de aprendizaje de los estudiantes desde su lengua materna, en este caso la lengua de señas colombiana, para luego generar estrategias de enseñanza, desde un diseño universal del aprendizaje estableciendo un contexto estratégico, y así ser parte de la formación de licenciados competentes. De la misma manera, es importante proporcionar el tiempo suficiente para que el estudiante pueda prepararse adecuadamente para la evaluación.

Es relevante a la hora de la planeación pedagógica tener en cuenta los componentes del aprendizaje estratégico que plantea Monereo (2007): aspectos cognitivos, metacognitivos y afectivos. Los aspectos cognitivos remiten a los procesos, habilidades, estrategias que facilitan el manejo de información, tanto generales (análisis, síntesis, analogías, etc.) como específicamente disciplinarios (para el caso de la matemática, se tienen los siguientes: inducción, deducción, demostración, graficación, factorización, etc.). Los aspectos metacognitivos remiten a la capacidad que tienen los seres humanos de estar conscientes de su actividad cognitiva propia, concurrentemente con la realización de alguna tarea intelectualmente exigente. Y los aspectos afectivos se refieren a las actitudes, emociones y creencias, tanto acerca de sí mismo, como en relación con la disciplina y con las demás personas.

De la misma manera es importante resaltar que cuando el individuo se ve enfrentado a una situación problemática, de manera innata e inmediata, toma la decisión de elaborar un plan de acción, y su cerebro, simula, casi que inmediatamente, las decisiones futuras y los resultados previsibles (consecuencias). Es así como el aprendizaje estratégico supone de una planificación contextual, para obtener un verdadero cambio cognitivo, actitudinal, procedimental y conceptual; y a su vez, requiere la activación consciente y condicionada de otros aprendizajes. El conocimiento estratégico puede compensar la escaces del conocimiento conceptual, y potenciar la eficacia del conocimiento procedimental (Monereo, 2007). 


\section{Referencias}

Blanco Guijarro, R. (2008). Marco conceptual sobre educación inclusiva. 48 a reunión de la Conferencia Internacional de Educación. 8. Ginebra.

Bernal, S. G., Pereira Alba, O. L., \& Rodríguez Jiménez, G. E. (2018). Comunicación Humana Interpersonal: Una mirada sistémica. Bogotá: ǏbērAM. Obtenido de http://repositorio.iberoamericana. edu.co/bitstream/001/596/1/Comunicaci\%C3\%B3n\%20humana\%20 interpersonal\%20una\%20mirada\%20sist\%C3\%A9mica.pdf

CAST (Center for Applied Special Technology). (2013). Pautas sobre el diseño Universal para el aprendizaje. Traducción al español version 2.0. Universal Design for Learning Guidelines version 2.0. Madrid. Obtenido de http://www.udlcenter.org/sites/udlcenter.org/files/UDL Guidelines v2.0-full espanol.docx

Castilla, C. A., Ayala Cardona, J., \& Acosta Pineda, D. J. (20 de Junio de 2015). Antecedentes en atención pedagógica a la población sorda: Una revisión contextual. Revista Iberoamericana de Psicología: Ciencia y tecnología, 8(1), 7 - 15

Congreso de Colombia. (9 de Agosto de 2005). Ley 982 de 2005. Bogotá cololmbia.

Dalmau Montalá, M., Guasch Murillo, D., Sala Bars, I., Llinares Fité, M., Dotras Ruscalleda, P., Álvarez Suau, M. H., \& Giné Giné, C. G. (2002). Diseño Universal para la Instrucción (DUI). Indicadores para su implementación en el ámbito universitario. Catalunya, España: Universidad Ramón Llull, Càtedra d’Accessibilitat de la Universitat Politècnica.

Díaz Barriga, F., \& Hernández Rojas, G. (2007). Estrategias docentes para un aprendizaje significativo una interpretación constructivista. México: Mc Graw Hill.

Díez Villoria, E., \& Sánchez Fuentes, S. (30 de Diciembre de 2014). Diseño universal para el aprendizaje como metodología docente para atender a la diversidad en la universidad. Aula Abierta, 43(2), 87-93. Recuperado el Octubre de 24 de 2018, de http://www.elsevier.es/ index.php?p=revista\&pRevista=pdf-simple\&pii=S0210277315000025 $\& r=389$

Fajardo, M. S. (2017). La Educación Superior Inclusiva en Algunos Países de Latinoamérica: Avances, Obstáculos y Retos. Revista Latinoamericana de Educación Inclusiva, 11(1), 171-197. Obtenido de https://dx.doi.org/10.4067/S0718-73782017000100011

Fiske, E., \& Peppler Barry, U. (2000). Foro Mundial sobre la Educación, Dakar, Senegal, 26-28 de abril de 2000: informe final. Documento de programa o reunión, UNESCO, Dakar.

Fontal González, A., \& Mejía Zuluaga, C. (2015). Construcción del conocimiento de las personas sordas: una aproximación a sus características socio- familiares. Informes Psicológicos, 15(2), 47-66. doi: http://dx.doi.org/10.18566/infpsicv15n2a03

Giné Giné, C., \& Font, J. (2007). El alumnado con discapacidad intelectual y del desarrollo. 879-914. España: Graó.

González, F. E. (2009). Metacognición y aprendizaje estratégico. Revista Integra Educativa, 127 - 136.

Hernández Sampieri, R., Fernández Collado, C., \& Baptista Lucio, M. (2014). Metodología de la Investigación, Sexta Edición. México, México, D.F MCGRAW-HILL / INTERAMERICANA EDITORES, S.A. DE C.V.

INSOR. (2006). Educación bilingüe para sordos - etapa escolar-. Orientaciones Pedagógicas. . Bogotá: Imprenta Nacional.

Marín Ortega , E., \& Moreno Hernández, A. (2007). Competencia para aprender a aprender. Alianza Editorial. Alianza.

Massone, M. I., Druetta, J. C., \& Simon, M. (2003). Arquitectura de la escuela de sordos. LibrosEnRed.

Ministerio de Educación Nacional - MEN. (2013). Lineamientos Política de educación superior inclusiva. Obtenido de Colombia Aprende: https://redes.colombiaaprende.edu.co/ntg/men/pdf/Lineamientos.pdf
Ministerio de Educación Nacional- MEN. (Marzo de 2008). Evaluación Anual de desempeño laboral a docentes y directivos. Guía mtodológica. Bogotá, Colombia. Obtenido de https://www. mineducacion.gov. co/1621/articles-157083 recurso 6.unknown

Monereo, C. (2000). El asesoramiento en el ámbito de las estrategias de aprendizaje. Visor, 15-62.

Monereo, C. (2007). El aprendizaje estratégico. Preguntas con respuesta. Perspectiva escolar, 2-10.

Monereo, C. (2007). Hacia un nuevo paradigma del aprendizaje estratégico: el papel de la mediación social, del self y de las emociones.

Electronic Journal of Research in Educational Psychology, 5(2), 497 - 534. Recuperado el Octubre de 23 de 2018, de https://www.redalyc. org/articulo.oa?id=293121946003

Morales García, A. M. (2015). El español escrito como segunda lengua en el sordo: apuntes para su enseñanza. Boletín de Lingüística, 27(43-44), 118-131.

OMS- Organización Mundial de la salud. (2011). Informe Mundial sobre la Discpacidad. Recuperado el 24 de Octubre de 2018, de. Obtenido de http://www.who.int/disabilities/world report/2011/summary es.pd

Opperti, R. (2008). Inclusión educativa: el camino del futuro. Un desafío para compartir. 48a reunión de la Conferencia Internacional de Educación CIE. 1. Ginebra.

Pastor, C. A., Sánchez Hípola, P., Sánchez Serrano , J., \& Zubillaga del Río, A. (Octubre de 2013). Club ensayos. Obtenido de https://www. clubensayos.com/Temas-Variados/Dise\%C3\%B1o-Universal-DelAprendizaje/2076770.html

Pizano Chavez, G. (2014). Las estrategias de aprendizaje y su relevancia en el rendimiento académico de los alumnos. Revista de Investigación Educativa, 27-30.

Román Sánchez, J. M., \& Gallego Rico, J. G. (2008). Escalas de estrategias. ACRA. Manual 4a edición. (T. E. S.A, Ed.) Madrid. Obtenido de http:// www.web.teaediciones.com/Ejemplos/ACRA extracto web.pdf

Román, J. M., \& Gallego, S. (1994). Escalas de estrategias. Madrid: PSEA.

Rué, J. (2010). El Aprendizaje Autónomo en Educación Superior. Madrid: Narcea, S. A. de Ediciones.

Ruiz, A. L. (2014). ¿Educación inclusiva? Análisis del marco jurídico sobre el derecho de acceso y permanencia en la educación superior para las personas Sordas en la ciudad de Bogotá. Derecho Público, 33. doi: http://dx.doi.org/10.15425/redepub.33.2014.18

Sabariego Puig, M., Vila, R., \& Sandín Esteban, M. P. (2014). El análisis cualitativo de datos con ATLAS.ti. Revista d'Innovació Recerca en Educació, 119-133.

Sandín, M. P. (2003). Investigación Cualitativa en Educación. Fundamentos y Tradiciones. Revista de Pedagogía, 26(77), 48-58.

Schorn, M. (2008). La conducta impulsiva del niño sordo. Argentina: Lugar.

Sverdlick, I., Ferrari, P., \& Jaimovich, A. (2005). Desigualdad e inclusión en la educación superior. Un estudio comparado en cinco países de latinoamérica. 9. Buenos Aires: Laboratorio de Políticas Públicas.

Taylor, S., \& Bogdan, R. (1987). Introducciona los Metodos Cualitativos de Investigacion. Barcelona: Paidós.

Unesco. (Octubre de 9 de 1998). Declaración mundial sobre Educación Superior en el siglo XXI: Visión y Acción. Obtenido de http://www. unesco.org/education/educprog/wche/declaration spa.htm

Unesco. (2009). Directrices sobre políticas de inclusión en la educación. Documento de programa o de reunión, París.

Varguillas, C. (2006). El uso de Atlas. Ti y la creatividad del investigador en el análisis cualitativo de contenido upel. Instituto. Laurus, 73-87.

Veinberg, S. (Febrero de 2007). Perspectiva Socioantropológica de la Sordera. Recuperado el 25 de Octubre de 2018, de http:// www.cultura-sorda.org/wp-content/uploads/2015/03/Veinberg perspectiva socioantropologica Sordera.pdf 
Estrategias de enseñanza aprendizaje en el proceso formativo de estudiantes sordos en la educación superior 